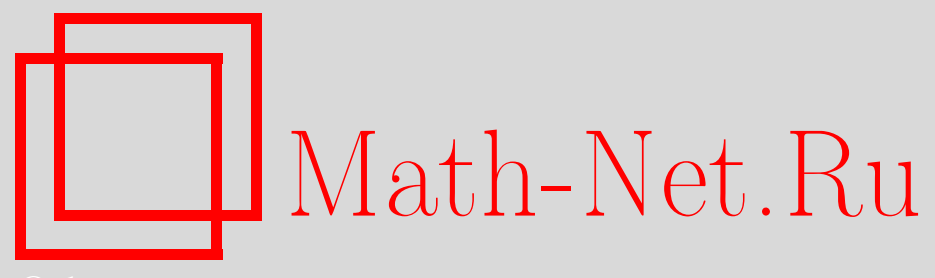

А. М. Зубков, В. И. Круглов, Моментные характеристики весов векторов в случайных двоичных линейных кодах, Матем. вопр. криптогр., 2012, том 3, выпуск 4, 55-70

DOI: https://doi.org/10.4213/mvk67

Использование Общероссийского математического портала Math-Net.Ru подразумевает, что вы прочитали и согласны с пользовательским соглашением http://www.mathnet.ru/rus/agreement

Параметры загрузки:

IP : 54.209 .52 .79

26 апреля 2023 г., 14:21:38

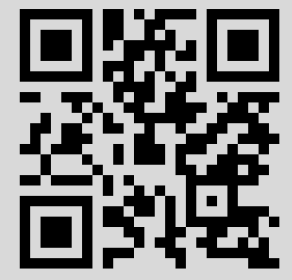




\title{
Моментные характеристики весов векторов в случайных двоичных линейных кодах
}

\author{
А. М. Зубков, В. И. Круглов \\ Математический институт им. В. А. Стеклова РАН, Москва
}

Получено 20.V.2011

Для случайных двоичных линейных кодов найдены формулы для первых моментов элементов весового спектра и явные выражения для типичных значений минимального веса ненулевых кодовых слов. Получены также верхние оценки вероятности того, что случайные независимые двоичные векторы с заданными количествами ненулевых элементов линейно зависимы.

Ключевые слова: случайные двоичные линейные коды, моменты весового спектра, минимальное ненулевое кодовое слово, линейная зависимость случайных векторов

\section{Moments of codeword weights in random binary linear codes}

\section{A. M.Zubkov, V. I. Kruglov}

Steklov Mathematical Institute of RAS, Moscow

\begin{abstract}
We consider random uniform binary linear codes. Formulas for the first moments of weight spectrum are obtained along with the explicit estimates for the typical values of the minimal weight of nonzero codewords. Upper bounds for the probability of linear dependence between independent random binary vectors with given weights are obtained also.

Key words: random binary linear codes, weight spectrum moments, minimal nonzero codeword, linear dependence of random vectors
\end{abstract}

Citation: Mathematical Aspects of Cryptography, 2012, vol. 3, no. 4, pp. 55-70 (Russian). 


\section{1. Введение}

В теории кодирования, дискретной геометрии, криптографии и других разделах математики рассматриваются линейные коды, т. е. линейные подпространства конечномерных пространств над конечными полями или кольцами. Важными характеристиками кодов являются их весовые спектры (количества кодовых слов с заданным весом, т.е. числом ненулевых координат) и минимальные расстояния между кодовыми словами (для линейных кодов они равны минимальному весу ненулевых кодовых слов).

В настоящей работе для случайных двоичных линейных кодов получены формулы для первых моментов элементов весового спектра, явные выражения для типичных значений минимального веса ненулевых кодовых слов. Получены также верхние оценки вероятности того, что случайные независимые двоичные векторы с заданными числами ненулевых элементов линейно зависимы.

\section{2. Обозначения}

Рассмотрим $N$-мерное линейное пространство $B^{N}=\mathrm{GF}(2)^{N}=\{X=$ $\left.=\left(x_{1}, \ldots, x_{N}\right): x_{1}, \ldots, x_{N} \in \mathrm{GF}(2)\right\}$. Под линейным кодом размерности $k$ будем понимать $k$-мерное подпространство $L \subset B^{N}$.

Весом двоичного вектора $X=\left(x_{1}, \ldots, x_{N}\right) \in B^{N}$ будем называть величину $w(X)=\sum_{i=1}^{N} I\left\{x_{i} \neq 0\right\}$, т. е. количество ненулевых координат в векторе $X$.

Через $B_{s}^{N}$ и $B_{\leq s}^{N}$ будем обозначать соответственно множество векторов фиксированного веса $S$ и множество ненулевых векторов веса, не превосходящего $s$, в $B^{N}$ :

$$
B_{s}^{N}=\left\{X \in B^{N} \mid w(X)=s\right\}, \quad B_{\leq s}^{N}=\left\{X \in B^{N} \mid 0<w(X) \leq s\right\} ;
$$

тогда $B^{N}=\bigsqcup_{s=0}^{N} B_{s}^{N}$ и $B_{\leq s}^{N}=\bigsqcup_{r=1}^{s} B_{r}^{N}$.

Пусть $v_{s}(L)=\left|L \cap B_{s}^{N}\right|$ и $v_{\leq s}(L)=\left|L \cap B_{\leq s}^{N}\right|-$ количество векторов веса $s$ и количество ненулевых векторов веса не больше $s$ в линейном коде $L$; набор $\left\{v_{s}(L)\right\}_{s=0}^{N}$ называют весовым спектром кода $L$.

Предельные пуассоновские теоремы для случайных величин $\mho_{S}(L)$ доказаны В.А.Копытцевым и В.Г. Михайловым в [9-12] при условии, что $N \rightarrow \infty, s / N \rightarrow 0$. Мы рассматриваем ситуацию, когда $N, k \rightarrow \infty, k / N \rightarrow c$, $c \in(0,1)$; показано, что при этих условиях распределение минимального веса ненулевого вектора сосредоточивается около величины $s$, удовлетворяющей соотношению $s / N \rightarrow c_{1}=c_{1}(c) \in(0,1)$, таким образом, теоремы из [9-12] к этому случаю не применимы. 


\section{3. Первые моменты случайной величины $v_{s}(L)$}

Утверждение 1. Количество упорядоченных наборов линейно независимых векторов $Z_{1}, \ldots, Z_{m}$ в k-мерном линейном двоичном коде равно

$$
\left(2^{k}-1\right)\left(2^{k}-2\right) \cdot \ldots \cdot\left(2^{k}-2^{m-1}\right) .
$$

Утверждение 2. Пусть $X_{1}, \ldots, X_{m}$ - фиксированные линейно независимые векторы в $k$-мерном линейном коде $L \subset B^{N}$. Количество способов их (упорядоченного) дополнения до базиса L равно

$$
\left(2^{k}-2^{m}\right) \cdot \ldots \cdot\left(2^{k}-2^{k-1}\right)
$$

Утверждение 3. Количество линейных k-мерных кодов в $B^{N}$ равно

$$
\frac{\left(2^{N}-1\right)\left(2^{N}-2^{1}\right) \ldots\left(2^{N}-2^{k-1}\right)}{\left(2^{k}-1\right)\left(2^{k}-2^{1}\right) \ldots\left(2^{k}-2^{k-1}\right)} .
$$

Эти утверждения хорошо известны, см., например, [14, п. 12.2], или [13, гл. $3, \S 4]$.

Лемма 1. Вероятность того, что случайный линейный код размерности $k$ в $B^{N}$ содержит фиксированные линейно независимые векторы $X_{1}, X_{2}, \ldots, X_{m} \in B^{N}$, равна

$$
\frac{\left(2^{k}-1\right)\left(2^{k}-2^{1}\right) \ldots\left(2^{k}-2^{m-1}\right)}{\left(2^{N}-1\right)\left(2^{N}-2^{1}\right) \ldots\left(2^{N}-2^{m-1}\right)} .
$$

Доказательство. Линейно независимые векторы $X_{1}, X_{2}, \ldots, X_{m} \in B^{N}$ однозначно определяют $m$-мерное линейное подпространство (линейный код) в $B^{N}$. Согласно утверждению 3 , существует

$$
M_{N, k}=\frac{\left(2^{N}-1\right)\left(2^{N}-2^{1}\right) \ldots\left(2^{N}-2^{k-1}\right)}{\left(2^{k}-1\right)\left(2^{k}-2^{1}\right) \ldots\left(2^{k}-2^{k-1}\right)}
$$

$k$-мерных линейных кодов в $B^{N}$, и каждый $k$-мерный код содержит

$$
M_{k, m}=\frac{\left(2^{k}-1\right)\left(2^{k}-2^{1}\right) \ldots\left(2^{k}-2^{m-1}\right)}{\left(2^{m}-1\right)\left(2^{m}-2^{1}\right) \ldots\left(2^{m}-2^{m-1}\right)}
$$


разных $m$-мерных линейных кодов. Поэтому число таких пар $\left(L^{\prime}, L^{\prime \prime}\right)$ линейных кодов в $B^{N}$, что $L^{\prime}-m$-мерный код, $L^{\prime \prime}-k$-мерный код и $L^{\prime} \subseteq L^{\prime \prime}$, равно

$$
M_{N, k} M_{k, m}=\frac{\left(2^{N}-1\right)\left(2^{N}-2^{1}\right) \ldots\left(2^{N}-2^{k-1}\right)}{\left(2^{m}-1\right)\left(2^{m}-2^{1}\right) \ldots\left(2^{m}-2^{m-1}\right)} \prod_{j=m}^{k-1} \frac{1}{2^{k}-2^{j}} .
$$

В силу симметрии каждый из

$$
M_{N, m}=\frac{\left(2^{N}-1\right)\left(2^{N}-2^{1}\right) \ldots\left(2^{N}-2^{m-1}\right)}{\left(2^{m}-1\right)\left(2^{m}-2^{1}\right) \ldots\left(2^{m}-2^{m-1}\right)}
$$

$m$-мерных кодов входит в

$$
\frac{M_{N, k} M_{k, m}}{M_{N, m}}=\frac{\frac{\left(2^{N}-1\right)\left(2^{N}-2^{1}\right) \ldots\left(2^{N}-2^{k-1}\right)}{\left(2^{m}-1\right)\left(2^{m}-2^{1}\right) \ldots\left(2^{m}-2^{m-1}\right)} \prod_{j=m}^{k-1} \frac{1}{2^{k}-2^{j}}}{\frac{\left(2^{N}-1\right)\left(2^{N}-2^{1}\right) \ldots\left(2^{N}-2^{m-1}\right)}{\left(2^{m}-1\right)\left(2^{m}-2^{1}\right) \ldots\left(2^{m}-2^{m-1}\right)}}=\prod_{j=m}^{k-1} \frac{2^{N}-2^{j}}{2^{k}-2^{j}}
$$

пар $\left(L^{\prime}, L^{\prime \prime}\right)$ как первая компонента. Значит, при фиксированном $L^{\prime}$ число вторых компонент этих пар (равное числу $k$-мерных кодов, содержащих $L^{\prime}$ ) вычисляется по формуле (1), а вероятность того, что равновероятно выбранный $k$-мерный код содержит данный $m$-мерный код $L^{\prime}$, равна

$$
\begin{gathered}
\frac{M_{N, k} M_{k, m}}{M_{N, m} M_{N, k}}=\frac{\left(2^{N}-2^{m}\right) \ldots\left(2^{N}-2^{k-1}\right)}{\left(2^{k}-2^{m}\right) \ldots\left(2^{k}-2^{k-1}\right)}: \frac{\left(2^{N}-1\right)\left(2^{N}-2^{1}\right) \ldots\left(2^{N}-2^{k-1}\right)}{\left(2^{k}-1\right)\left(2^{k}-2^{1}\right) \ldots\left(2^{k}-2^{k-1}\right)}= \\
=\frac{\left(2^{k}-1\right)\left(2^{k}-2^{1}\right) \ldots\left(2^{k}-2^{m-1}\right)}{\left(2^{N}-1\right)\left(2^{N}-2^{1}\right) \ldots\left(2^{N}-2^{m-1}\right)} \cdot \quad
\end{gathered}
$$

Теорема 1. Если $L$ - случайный k-мерный код в $B^{N}$, имеющзий равновероятное распределение на множестве всех таких кодов, то при $s=1, \ldots, N$

$$
\begin{gathered}
\mathbf{E} v_{s}(L)=C_{N}^{s} \frac{2^{k}-1}{2^{N}-1} \\
\mathbf{D} v_{s}(L)=C_{N}^{s} \frac{\left(2^{k}-1\right)}{\left(2^{N}-1\right)} \frac{\left(2^{N}-2^{k}\right)}{\left(2^{N}-2\right)}\left(1-\frac{C_{N}^{s}}{2^{N}-1}\right)
\end{gathered}
$$

u при $s, t \in\{1, \ldots, N\}, s \neq t$,

$$
\operatorname{cov}\left(v_{s}(L), v_{t}(L)\right)=-C_{N}^{s} C_{N}^{t} \frac{\left(2^{k}-1\right)\left(2^{N}-2^{k}\right)}{\left(2^{N}-1\right)^{2}\left(2^{N}-2\right)} .
$$


ЗАМЕчАНИЕ. Согласно теореме 1 , при $s \neq t$ коэффициент корреляции

$$
\operatorname{corr}\left(v_{s}(L), v_{t}(L)\right)=-\frac{\sqrt{C_{N}^{s} C_{N}^{t}}}{2^{N}-1} \sqrt{\left(1-\frac{C_{N}^{s}}{2^{N}-1}\right)\left(1-\frac{C_{N}^{t}}{2^{N}-1}\right)}
$$

отрицателен и по абсолютной величине при любых $s \neq t$ меньше $2^{-N} C_{N}^{[N / 2]} \approx$ $\approx \sqrt{\frac{2}{\pi N}}$, кроме того, коэффициент корреляции (как и $\left.\mathbf{E} v_{s}(L), \mathbf{E} v_{t}(L)\right)$ быстро уменьшается, когда $s$ и/или $t$ удаляются от $N / 2$.

Если бы индикаторы $I_{X}=I\{X \in L\}, X \in B^{N}$, были независимыми с $\mathbf{P}\{X \in L\}=\frac{2^{k}-1}{2^{N}-1}, X \in B^{N}$, то дисперсия суммы $\sum_{X: w(X)=s} I_{X}$, т. е. дисперсия числа кодовых слов веса $s$, была бы равна

$$
C_{N}^{S} \frac{2^{k}-1}{2^{N}-1}\left(1-\frac{2^{k}-1}{2^{N}-1}\right)=C_{N}^{S} \frac{\left(2^{k}-1\right)\left(2^{N}-2^{k}\right)}{\left(2^{N}-1\right)^{2}}=\left(1-\frac{C_{N}^{s}-1}{2^{N}-2}\right) \mathbf{D} v_{S}(L) .
$$

Все это показывает, что попарная зависимость между событиями $\{X \in L\}$, $X \in B^{N}$, очень слаба. лемме 1 ,

Доказательство. Так как $v_{S}(L)=\sum_{X \in B_{s}^{N}} I\{X \in L\}$, то, согласно

$$
\mathbf{E} v_{S}(L)=\sum_{X \in B_{s}^{N}} \mathbf{P}\{X \in L\}=\sum_{X \in B_{s}^{N}} \frac{2^{k}-1}{2^{N}-1}=C_{N}^{s} \frac{2^{k}-1}{2^{N}-1},
$$

и для любых двух различных ненулевых векторов $X_{1}, X_{2} \in B^{N}$

$$
\begin{aligned}
\operatorname{cov}\left(I\left\{X_{1} \in L\right\},\right. & \left.I\left\{X_{2} \in L\right\}\right)= \\
= & \mathbf{E} I\left\{X_{1}, X_{2} \in L\right\}-\mathbf{E} I\left\{X_{1} \in L\right\} \mathbf{E} I\left\{X_{2} \in L\right\}= \\
& =\frac{\left(2^{k}-1\right)\left(2^{k}-2\right)}{\left(2^{N}-1\right)\left(2^{N}-2\right)}-\frac{\left(2^{k}-1\right)^{2}}{\left(2^{N}-1\right)^{2}}=-\frac{\left(2^{k}-1\right)\left(2^{N}-2^{k}\right)}{\left(2^{N}-1\right)^{2}\left(2^{N}-2\right)} .
\end{aligned}
$$

Поэтому

$$
\begin{gathered}
\mathbf{D} v_{s}(L)=\sum_{X \in B_{s}^{N}} \mathbf{D} I\{X \in L\}+\sum_{\substack{X_{1}, X_{2} \in B_{s}^{N} \\
X_{1} \neq X_{2}}} \operatorname{cov}\left(I\left\{X_{1} \in L\right\}, I\left\{X_{2} \in L\right\}\right)= \\
=C_{N}^{S} \frac{2^{k}-1}{2^{N}-1}\left(1-\frac{2^{k}-1}{2^{N}-1}\right)-C_{N}^{S}\left(C_{N}^{s}-1\right) \frac{\left(2^{k}-1\right)\left(2^{N}-2^{k}\right)}{\left(2^{N}-1\right)^{2}\left(2^{N}-2\right)}=
\end{gathered}
$$




$$
\begin{gathered}
=C_{N}^{s} \frac{\left(2^{k}-1\right)\left(2^{N}-2^{k}\right)}{\left(2^{N}-1\right)^{2}}\left(1-\frac{C_{N}^{s}-1}{2^{N}-2}\right)= \\
=C_{N}^{s} \frac{\left(2^{k}-1\right)\left(2^{N}-2^{k}\right)}{\left(2^{N}-1\right)\left(2^{N}-2\right)}\left(1-\frac{C_{N}^{s}}{2^{N}-1}\right) .
\end{gathered}
$$

Наконец, при $1 \leq s<t \leq N$ в силу линейности оператора ковариации

$$
\begin{aligned}
& \operatorname{cov}\left(v_{s}(L), v_{t}(L)\right)=\operatorname{cov}\left(\sum_{X \in B_{s}^{N}} I\{X \in L\}, \sum_{X \in B_{t}^{N}} I\{X \in L\}\right)= \\
& =\sum_{X_{1} \in B_{s}^{N}} \sum_{X_{2} \in B_{t}^{N}} \operatorname{cov}\left(I\left\{X_{1} \in L\right\}, I\left\{X_{2} \in L\right\}\right)=-C_{N}^{S} C_{N}^{t} \frac{\left(2^{k}-1\right)\left(2^{N}-2^{k}\right)}{\left(2^{N}-1\right)^{2}\left(2^{N}-2\right)} .
\end{aligned}
$$

\section{4. Первые моменты случайной величины $v_{\leq s}(L)$ и оценки минимального веса}

Теорема 2. Для случайного равновероятного линейного k-мерного кода $L$ в $B^{N}$ nри $s=1, \ldots, N$

$$
\begin{gathered}
\mathbf{E} v_{\leq s}(L)=\frac{2^{k}-1}{2^{N}-1} \sum_{r=1}^{s} C_{N}^{r}, \\
\mathbf{D} v_{\leq s}(L)=\frac{2^{N}-2^{k}}{2^{N}-2}\left(1-\frac{1}{2^{N}-1} \sum_{r=1}^{s} C_{N}^{r}\right) \frac{2^{k}-1}{2^{N}-1} \sum_{r=1}^{s} C_{N}^{r} \leq \frac{2^{N}-2^{k}}{2^{N}-2} \mathbf{E} v_{\leq s}(L) .
\end{gathered}
$$

Доказательство. Используя теорему 1, находим:

$$
\mathbf{E} v_{\leq s}(L)=\sum_{r=1}^{s} \mathbf{E} v_{r}(L)=\frac{2^{k}-1}{2^{N}-1} \sum_{r=1}^{s} C_{N}^{r} .
$$

Далее,

$$
\begin{gathered}
\mathbf{D} v_{\leq s}(L)=\mathbf{D} \sum_{r=1}^{s} v_{r}(L)=\sum_{r=1}^{s} \mathbf{D} v_{r}(L)+\sum_{\substack{r_{1}, r_{2}=1 \\
r_{1} \neq r_{2}}}^{s} \operatorname{cov}\left(v_{r_{1}}(L), v_{r_{2}}(L)\right)= \\
=\sum_{r=1}^{s} C_{N}^{r} \frac{\left(2^{k}-1\right)\left(2^{N}-2^{k}\right)}{\left(2^{N}-1\right)\left(2^{N}-2\right)}\left(1-\frac{C_{N}^{r}}{2^{N}-1}\right)-\sum_{\substack{r_{1}, r_{2}=1 \\
r_{1} \neq r_{2}}}^{s} C_{N}^{r_{1}} C_{N}^{r_{2}} \frac{\left(2^{k}-1\right)\left(2^{N}-2^{k}\right)}{\left(2^{N}-1\right)^{2}\left(2^{N}-2\right)}= \\
=\frac{\left(2^{k}-1\right)\left(2^{N}-2^{k}\right)}{\left(2^{N}-1\right)\left(2^{N}-2\right)}\left(1-\frac{1}{2^{N}-1} \sum_{r=1}^{s} C_{N}^{r}\right) \sum_{r=1}^{s} C_{N}^{r} . \quad \square
\end{gathered}
$$


Следствие 1. Если $L \subset B^{N}-$ случайный равновероятный $k$-мерный код $и \mu(L)=\min \{\varpi(x): x \in L \backslash\{0\}\}$, то

$$
\frac{1}{1+\frac{2^{N}-2^{k}}{2^{N}-2}\left(\mathbf{E} v_{\leq s}(L)\right)^{-1}} \leq \mathbf{P}\{\mu(L) \leq s\} \leq \mathbf{E} v_{\leq s}(L) .
$$

Доказательство. Используем равенство $\{\mu(L) \leq s\}=\left\{v_{\leq s}(L) \geq 1\right\}$, теорему 2 и известные неравенства для целочисленной неотрицательной случайной величины $\xi$ (нижняя оценка - следствие неравенства $\left.(\mathbf{E}\{\xi \mid \xi \geq 1\})^{2} \leq \mathbf{E}\left\{\xi^{2} \mid \xi \geq 1\right\}\right)$ :

$$
\frac{(\mathbf{E} \xi)^{2}}{\mathbf{D} \xi+(\mathbf{E} \xi)^{2}}=\frac{(\mathbf{E} \xi)^{2}}{\mathbf{E} \xi^{2}} \leq \mathbf{P}\{\xi \geq 1\} \leq \mathbf{E} \xi
$$

Правое неравенство следствия 1 очевидно, а левое получается несложными преобразованиями:

$$
\begin{aligned}
\frac{\left(\mathbf{E} v_{\leq s}(L)\right)^{2}}{\mathbf{D} v_{\leq s}(L)+\left(\mathbf{E} v_{\leq s}(L)\right)^{2}}=\frac{1}{1+\mathbf{D} v_{\leq s}(L) /\left(\mathbf{E} v_{\leq s}(L)\right)^{2}} \geq & \\
& \geq \frac{1}{1+\frac{2^{N}-2^{k}}{2^{N}-2}\left(\mathbf{E} v_{\leq s}(L)\right)^{-1}} .
\end{aligned}
$$

Нижняя оценка следствия приближается к 1 с ростом $\mathbf{E} v_{\leq s}(L)$, поэтому распределение минимального веса ненулевого вектора случайного равновероятного $k$-мерного линейного кода в $\mathrm{GF}(2)^{N}$ сосредоточено вблизи значения $s=s_{0}$, при котором $\mathbf{E} v_{\leq s}(L)$ становится больше 1, т. е.

$$
\sum_{r=1}^{s_{0}-1} C_{N}^{r}<2^{N-k} \leq \sum_{r=1}^{s_{0}} C_{N}^{r} ;
$$

действительно, в области $s<s_{0}$ верхняя оценка вероятности $\mathbf{P}\{\mu(L) \leq s\}$ меньше 1 и убывает при уменьшении $s$, а в области $s>s_{0}$ нижняя оценка больше $\frac{1}{2}$ и приближается к 1 при возрастании $s$.

Асимптотические формулы, связывающие $N, k$ и $s_{0}$, приведены в теореме 1 статьи [4]. Явные оценки для формул в теореме 2 и в следствии 1 , не содержащие сумм биномиальных коэфициентов, можно вывести с помощью приведенных в $[5,6]$ переформулировок некоторых результатов статьи [2] об оценках хвостов биномиального распределения. Частным случаем 
этих результатов являются справедливые при $0 \leq s<N / 2$ неравенства

$$
2^{N} \Phi\left(-\sqrt{N V\left(1-\frac{2 s}{N}\right)}\right) \leq \sum_{r=0}^{s} C_{N}^{r} \leq 2^{N} \Phi\left(-\sqrt{N V\left(1-\frac{2(s+1)}{N}\right)}\right),
$$

где $\Phi(x)=\frac{1}{\sqrt{2 \pi}} \int_{-\infty}^{x} e^{-\frac{u^{2}}{2}} d u-$ функция стандартного нормального распределения и при $|z|<1$

$V(z)=(1-z) \ln (1-z)+(1+z) \ln (1+z)=\sum_{k=1}^{\infty} \frac{z^{2 k}}{k(2 k-1)}=z^{2}+\frac{z^{4}}{6}+\frac{z^{6}}{15}+\ldots$

Обозначая $2^{-N} \sum_{r=0}^{s} C_{N}^{r}$ через $\beta$, можно решить неравенства относительно $s$, используя функции $V^{-1}$ и $\Phi^{-1}$, обратные к $V$ и $\Phi$ :

$$
s \leq \frac{N}{2}\left(1-V^{-1}\left(\frac{1}{N}\left(\Phi^{-1}(\beta)\right)^{2}\right)\right) \leq s+1 .
$$

Если

$$
\sum_{r=1}^{s_{0}-1} C_{N}^{r}<2^{N-k} \leq \sum_{r=1}^{s_{0}} C_{N}^{r},
$$

то ввиду убывания функции $\left(\Phi^{-1}(x)\right)^{2}$ на $\left(0, \frac{1}{2}\right]$ и возрастания $V^{-1}(x)$ на $(0,1)$

$$
\begin{gathered}
s_{0}-1 \leq \frac{N}{2}\left(1-V^{-1}\left(\frac{1}{N}\left(\Phi^{-1}\left(2^{-N} \sum_{r=1}^{s_{0}-1} C_{N}^{r}\right)\right)^{2}\right)\right) \leq \\
\leq \frac{N}{2}\left(1-V^{-1}\left(\frac{1}{N}\left(\Phi^{-1}\left(2^{-k}\right)\right)^{2}\right)\right) \leq \\
\leq \frac{N}{2}\left(1-V^{-1}\left(\frac{1}{N}\left(\Phi^{-1}\left(2^{-N} \sum_{r=1}^{s_{0}} C_{N}^{r}\right)\right)^{2}\right)\right) \leq s_{0}+1,
\end{gathered}
$$

T. e.

$$
\left|s_{0}-\frac{N}{2}\left(1-V^{-1}\left(\frac{1}{N}\left(\Phi^{-1}\left(2^{-k}\right)\right)^{2}\right)\right)\right| \leq 1 .
$$

Отметим, что

$$
V^{-1}(z)=\sqrt{z}\left(1-\frac{z}{12}-\frac{13 z^{2}}{1440}-\frac{97 z^{3}}{40320}-\frac{25307 z^{4}}{29030400}-\ldots\right)
$$


и (см., например, [1]) при $0<\beta<\frac{1}{2}$ приближенная формула

$$
\Phi^{-1}(\beta) \approx-t_{\beta}+\frac{2.30753+0.27061 t_{\beta}}{1+0.99229 t_{\beta}+0.04481 t_{\beta}^{2}}, \quad t_{\beta}=\sqrt{-2 \ln \beta},
$$

имеет погрешность не более 0.003 .

Неравенство (3) уточняет асимптотические соотношения из [4].

Используя первые члены формул (4) и (5) как грубые приближения, нетрудно найти, что (если $N, k \rightarrow \infty$ и отношение $\frac{k}{N}$ отделено от 0 и 1 )

$$
s_{0} \approx \frac{N}{2}\left(1-\sqrt{\frac{2 k \ln 2}{N}}\right)=\frac{N}{2}-\sqrt{\frac{k N \ln 2}{2}}
$$

\section{5. Характеристики распределения суммы векторов с заданными весами}

Теорема 3. Если $X$ и $Y$ - независимые случайные векторы из $B^{N}$, причем $X$ имеет равномерное распределение на $B_{s}^{N}$, а $Y$ имеет равномерное распределение на $B_{t}^{N}$, то при $|s-t| \leq m \leq \min \{s+t, N\}$

$$
\mathbf{P}\{w(X \oplus Y)=m\}=p^{(N)}(t, s, m) \stackrel{\text { def }}{=} \frac{C_{s}^{\frac{t+s-m}{2}} C_{N-s}^{\frac{t-s+m}{2}}}{C_{N}^{t}} I\{m \equiv t+s(\bmod 2)\}
$$

$u$

$$
\mathbf{E} w(X \oplus Y)=s+t-\frac{2 s t}{N}, \quad \mathbf{D} w(X \oplus Y)=4 \frac{s(N-s) t(N-t)}{N^{2}(N-1)} .
$$

Следствие 2. Если $X$ и $Y$ - независимые случайные векторы из $B^{N}$, причем $X$ имеет равномерное распределение на $B_{s}^{N}$, а $Y$ имеет распределение, инвариантное относительно перестановок координат, и $\mathbf{P}\{w(Y)=j\}=q_{j}$, $j=0,1, \ldots, N, \boldsymbol{q}=\left(q_{0}, \ldots, q_{N}\right)$, mo

$$
\begin{gathered}
\mathbf{P}\{w(X \oplus Y)=m\}=p_{\boldsymbol{q}}^{(N)}(s, m) \stackrel{\text { def }}{=} \\
\stackrel{\text { def }}{=} \sum_{j=0}^{N} q_{j} \frac{C_{s}^{\frac{j+s-m}{2}} C_{N-s}^{\frac{j-s+m}{2}}}{C_{N}^{t}} I\{m \equiv j+s(\bmod 2)\} .
\end{gathered}
$$


Доказательство. Пусть $X=\left(x_{1}, \ldots, x_{N}\right), Y=\left(y_{1}, \ldots, y_{N}\right)-$ векторы из условия теоремы; тогда

$$
w(X \oplus Y)=w(X)+w(Y)-2\left|\left\{k \in\{1, \ldots, N\}: x_{k}=y_{k}=1\right\}\right| .
$$

Поэтому четность тогда и только тогда, когда

$$
\left|\left\{k \in\{1, \ldots, N\}: x_{k}=y_{k}=1\right\}\right|=\frac{s+t-m}{2} .
$$

Формула (6) следует из того, что число разных пар $X, Y \in B^{N} \mathrm{c} w(X)=s$, $w(Y)=t$ равно $C_{N}^{s} C_{N}^{t}$, а число таких пар векторов, имеющих ровно $r$ общих единичных координат, равно $C_{N}^{s} C_{S}^{r} C_{N-s}^{t-r}$.

Формулы для моментов вычислением с использованием равенств

$$
\begin{gathered}
\sum_{k \geq 0} C_{s}^{k} C_{N-s}^{t-k}=C_{N}^{t}, \quad \sum_{k \geq 0} k C_{s}^{k} C_{N-s}^{t-k}=s \sum_{k \geq 0} C_{s-1}^{k-1} C_{N-s}^{t-k}=s C_{N-1}^{t-1}, \\
\sum_{k \geq 0} k^{2} C_{s}^{k} C_{N-s}^{t-k}=\sum_{k \geq 0}(k(k-1)+k) C_{s}^{k} C_{N-s}^{t-k}= \\
=s(s-1) \sum_{k \geq 0} C_{s-2}^{k-2} C_{N-s}^{t-k}+s C_{N-1}^{t-1}=s(s-1) C_{N-2}^{t-2}+s C_{N-1}^{t-1} .
\end{gathered}
$$

Действительно,

$$
\begin{gathered}
\mathbf{E} w(X \oplus Y)=\frac{1}{C_{N}^{t}} \sum_{m \geq 0} m C_{s}^{\frac{t+s-m}{2}} C_{N-s}^{\frac{t-s+m}{2}}=\frac{1}{C_{N}^{t}} \sum_{k \geq 0}(s+t-2 k) C_{s}^{k} C_{N-s}^{t-k}= \\
=s+t-\frac{2 s}{C_{N}^{t}} C_{N-1}^{t-1}=s+t-\frac{2 s t}{N} \\
\mathbf{E} w^{2}(X \oplus Y)=\frac{1}{C_{N}^{t}} \sum_{m \geq 0} m^{2} C_{s}^{\frac{t+s-m}{2}} C_{N-s}^{\frac{t-s+m}{2}}=\frac{1}{C_{N}^{t}} \sum_{k \geq 0}(s+t-2 k)^{2} C_{s}^{k} C_{N-s}^{t-k}= \\
=(s+t)^{2}-2(s+t) \frac{2 s t}{N}+\frac{4}{C_{N}^{t}}\left(s(s-1) C_{N-2}^{t-2}+s C_{N-1}^{t-1}\right) \\
\mathbf{D} w^{2}(X \oplus Y)=\mathbf{E} w^{2}(X \oplus Y)-(\mathbf{E} w(X \oplus Y))^{2}= \\
=4\left(\frac{s(s-1) t(t-1)}{N(N-1)}+\frac{s t}{N}\right)-\left(\frac{2 s t}{N}\right)^{2}= \\
=4 s t \frac{N(s-1)(t-1)+N(N-1)-(N-1) s t}{N^{2}(N-1)}=4 \frac{s(N-s) t(N-t)}{N^{2}(N-1)} .
\end{gathered}
$$


Пусть $X_{1}, X_{2}, \ldots, X_{n} \in B^{N}$. Положим для любых $s \in\{0,1, \ldots, N\}$ и $k \in$ $\in\{1, \ldots, n\}$

$$
v_{s}^{*}\left(X_{1}, \ldots, X_{k}\right)=\sum_{a_{1}, \ldots, a_{k}=0}^{1} I\left\{w\left(\sum_{j=1}^{k} a_{j} X_{j}\right)=s\right\} .
$$

Если векторы $X_{1}, \ldots, X_{n}$ линейно независимы, то $\left\{v_{s}^{*}\left(X_{1}, \ldots, X_{n}\right)\right\}_{s=0}^{N}-$ весовой спектр линейного кода, порожденного набором $\left\{X_{1}, \ldots, X_{n}\right\}$. Величина $v_{0}^{*}\left(X_{1}, \ldots, X_{n}\right)$ равна числу линейных комбинаций векторов набора $\left\{X_{1}, \ldots, X_{n}\right\}$, равных 0 , т. е. она равна $2^{k}$, где $k=n-\operatorname{dim}\left\langle X_{1}, \ldots, X_{n}\right\rangle-$ разность между $n$ и размерностью линейного пространства, порожденного векторами $X_{1}, \ldots, X_{n}$. Все значения $v_{s}^{*}\left(X_{1}, \ldots, X_{n}\right)$ кратны $v_{0}^{*}\left(X_{1}, \ldots, X_{n}\right)$.

Теорема 4. Если $\left\{X_{1}, \ldots, X_{n}\right\}-$ набор независимых случайных векторов из $B^{N}$, в котором $X_{k}$ имеет равномерное распределение на множестве векторов с $\left(X_{k}\right)=s_{k}, k=1, \ldots, n$, то вектор-столбеи

$$
V_{n} \stackrel{\text { def }}{=}\left(\mathbf{E} v_{0}^{*}\left(X_{1}, \ldots, X_{n}\right), \mathbf{E} v_{1}^{*}\left(X_{1}, \ldots, X_{n}\right), \ldots, \mathbf{E} v_{N}^{*}\left(X_{1}, \ldots, X_{n}\right)\right)^{\top}
$$

вычисляется по формуле

$$
V_{n}=2^{n} P_{s_{n}}^{(N)} P_{s_{n-1}}^{(N)} \ldots P_{s_{1}}^{(N)}(1,0, \ldots, 0)^{\top},
$$

где $P_{s}^{(N)}=\left\|\frac{1}{2} p^{(N)}(s, i, j)+\frac{1}{2} \delta_{i, j}\right\|_{i, j=0}^{N}$, а величины $p^{(N)}(s, i, j)$ выччсляются по формуле (6).

Следствие 3. Если $\left\{\widetilde{X}_{1}, \ldots, \widetilde{X}_{n}\right\}$ - набор независимых случайных векторов из $B^{N}$, распределения которых инвариантны относительно перестановок координат, $u$

$$
\mathbf{P}\left\{w\left(\widetilde{X}_{k}\right)=j\right\}=q_{j}^{(k)}, \quad j \in\{0,1, \ldots, N\}, k=1,2, \ldots, n,
$$

то вектор-столбеи

$$
\widetilde{V}_{n} \stackrel{\text { def }}{=}\left(\mathbf{E} v_{0}^{*}\left(\widetilde{X}_{1}, \ldots, \widetilde{X}_{n}\right), \ldots, \mathbf{E} v_{N}^{*}\left(\widetilde{X}_{1}, \ldots, \widetilde{X}_{n}\right)\right)^{\top}
$$

вычисляется по формуле

$$
\widetilde{V}_{n}=2^{n} P_{\boldsymbol{q}^{(n)}}^{(N)} P_{\boldsymbol{q}^{(n-1)}}^{(N)} \ldots P_{\boldsymbol{q}^{(1)}}^{(N)}(1,0, \ldots, 0)^{\top},
$$


где $\boldsymbol{q}^{(k)}=\left(q_{0}^{(k)}, q_{1}^{(k)}, \ldots, q_{N}^{(k)}\right), P_{\boldsymbol{q}^{(k)}}^{(N)}=\left\|\frac{1}{2} p_{\boldsymbol{q}^{(k)}}^{(N)}(i, j)+\frac{1}{2} \delta_{i, j}\right\|_{i, j=0}^{N}$, а величинь $p_{\boldsymbol{q}^{(k)}}^{(N)}(i, j)$ вычисляются по формуле $(7)$.

ЗАМЕЧАНИЕ. В соответствии с замечанием, сделанным перед теоремой 4 , первые компоненты векторов $V_{n}$ в теореме 4 и $\widetilde{V}_{n}$ в следствии 3 равны математическому ожиданию числа линейных комбинаций, равных 0 ; поэтому они являются верхними оценками для увеличенной на 1 вероятности того, что векторы $X_{1}, \ldots, X_{n}$ (соответственно, $\widetilde{X}_{1}, \ldots, \widetilde{X}_{n}$ ) линейно зависимы, например:

$$
\mathbf{E} v_{0}^{*}\left(X_{1}, \ldots, X_{n}\right) \geq 1+\mathbf{P}\left\{X_{1}, \ldots, X_{n} \text { линейно зависимы }\right\} .
$$

Первые предельные теоремы для распределения ранга случайных булевых матриц с независимыми элементами были доказаны в статьях И. Н. Коваленко [8], М. В. Козлова [7], Г. В. Балакина [3].

Доказательство. Пусть $\varepsilon_{1}, \ldots, \varepsilon_{n}-$ независимые случайные индикаторы, $\mathbf{P}\left\{\varepsilon_{j}=0\right\}=\mathbf{P}\left\{\varepsilon_{j}=1\right\}=\frac{1}{2}$. Используя представление (8), находим

$$
\begin{gathered}
\mathbf{E} v_{s}^{*}\left(X_{1}, \ldots, X_{k}\right)= \\
=\mathbf{E} \sum_{a_{1}, \ldots, a_{k}=0}^{1} I\left\{w\left(\sum_{j=1}^{k} a_{j} X_{j}\right)=s\right\}=2^{k} \mathbf{P}\left\{w\left(\sum_{j=1}^{k} \varepsilon_{j} X_{j}\right)=s\right\} .
\end{gathered}
$$

Так как векторы $X_{1}, X_{2}, \ldots$ по условию независимы и их распределения инвариантны относительно перестановок координат, то при любых $k \geq 1$ и $a_{1}, a_{2}, \ldots \in \mathrm{GF}(2)$ распределение суммы $\sum_{j=1}^{k-1} a_{j} X_{j}$ инвариантно относительно перестановок координат. Значит, мы можем использовать равенство (6) в формуле полной вероятности:

$$
\begin{gathered}
r_{k}^{(N)}(s) \stackrel{\text { def }}{=} \mathbf{P}\left\{w\left(\sum_{j=1}^{k} \varepsilon_{j} X_{j}\right)=s\right\}= \\
=\sum_{m=0}^{N} \mathbf{P}\left\{w\left(\sum_{j=1}^{k} \varepsilon_{j} X_{j}\right)=s \mid w\left(\sum_{j=1}^{k-1} \varepsilon_{j} X_{j}\right)=m\right\} r_{k-1}^{(N)}(m)= \\
=\frac{1}{2} \sum_{m=0}^{N} p^{(N)}\left(s_{k}, m, s\right) r_{k-1}^{(N)}(m)+\frac{1}{2} \delta_{m, s} r_{k-1}^{(N)}(m),
\end{gathered}
$$


где $\delta_{m, s}=I\{m=s\}$ - дельта Кронекера. Это рекуррентное соотношение можно переписать в матричном виде, если обозначить через $R_{k}^{(N)}$ векторстолбец $\left(r_{k}^{(N)}(0), r_{k}^{(N)}(1), \ldots, r_{k}^{(N)}(N)\right)^{\top}$ :

$$
R_{k}^{(N)}=P_{s_{k}}^{(N)} R_{k-1}^{(N)}, \quad P_{s}^{(N)}=\left\|\frac{1}{2} p^{(N)}(s, i, j)+\frac{1}{2} \delta_{i, j}\right\|_{i, j=0}^{N} .
$$

Осталось заметить, что при $k=1$ равенство (9) выполняется, если положить $R_{0}^{(N)}=(1,0, \ldots, 0)^{\top} \in\{0,1\}^{N+1}$; после этого утверждение теоремы доказывается методом математической индукции.

Укажем еще один подход к получению верхней оценки вероятности наличия линейной зависимости между независимыми случайными векторами. Этот подход, как и описанный выше, пригоден для любых распределений случайных векторов, инвариантных относительно перестановок координат, однако мы рассмотрим только случай, когда все векторы имеют один и тот же вес, чтобы не загромождать формулы.

Теорема 5. Пусть $s \in\{1, \ldots, N-1\}$ и $X_{1}, \ldots, X_{n} \in B^{N}-$ независимые случайные векторы, которые имеют равновероятное распределение на множестве $\left\{X \in B^{N}: w(X)=s\right\}$. Обозначим $F=\left\{X_{1}, \ldots, X_{n}\right.$ линейно зависимы $\},$ тогда

$$
\mathbf{P}\{F\} \leq \frac{1}{2^{N}} \sum_{t=0}^{N} C_{N}^{t}\left[\left(1+\frac{c_{N, s, t}}{C_{N}^{s}}\right)^{n}-n \frac{C_{N, s, t}}{C_{N}^{s}}-1\right],
$$

где $c_{N, s, t}=\sum_{j \geq 0}(-1)^{j} C_{t}^{j} C_{N-t}^{s-j}$.

ЗАмечАниЕ. Так как $\left|c_{N, s, t}\right|=\left|\sum_{j \geq 0}(-1)^{j} C_{t}^{j} C_{N-t}^{s-j}\right|<\sum_{j \geq 0} C_{t}^{j} C_{N-t}^{s-j}=C_{N}^{s}$, то $\left|\frac{c_{N, s, t}}{C_{N}^{s}}\right|<1$.

Доказательство. Для каждого ненулевого вектора $v=\left(v_{1}, \ldots, v_{n}\right) \in$ $\in B^{n}$ рассмотрим событие $F_{v}=\left\{v_{1} X_{1} \oplus v_{2} X_{2} \oplus \ldots \oplus v_{n} X_{n}=0\right\}$. Тогда

$$
\begin{aligned}
\mathbf{P}\{F\} & =\mathbf{P}\left(\bigcup_{v \in B^{n} \backslash\{0\}} F_{v}\right) \leq \sum_{v \in B^{n} \backslash\{0\}} \mathbf{P}\left(F_{v}\right)= \\
& =\sum_{1 \leq r \leq n} C_{n}^{r} \mathbf{P}\left\{X_{1} \oplus \ldots \oplus X_{r}=0\right\}=\sum_{2 \leq r \leq n} C_{n}^{r} \mathbf{P}\left\{X_{1} \oplus \ldots \oplus X_{r}=0\right\},
\end{aligned}
$$

поскольку событие $\left\{X_{1} \oplus \ldots \oplus X_{r}=0\right\}$ является невозможным при $r=1$. 
Для вычисления вероятности $\mathbf{P}\left\{X_{1} \oplus \ldots \oplus X_{r}=0\right\}$ при $r \geq 2$ воспользуемся следующим фактом.

Лемма 2. Пусть случайные величины $\xi_{1}, \ldots, \xi_{m}$ принимают целочисленные значения, $\mathbf{P}\left\{\left(\xi_{1}, \ldots, \xi_{m}\right)=\left(k_{1}, \ldots, k_{m}\right)\right\}=p_{k_{1}, \ldots, k_{m}}, u$

$$
f\left(z_{1}, \ldots, z_{m}\right)=\mathbf{E} z_{1}^{\xi_{1}} \ldots z_{m}^{\xi_{m}}=\sum_{k_{1}, \ldots, k_{m} \in \mathbb{Z}} p_{k_{1}, \ldots, k_{m}} z_{1}^{k_{1}} \cdot \ldots \cdot z_{m}^{k_{m}}
$$

- производящуая функция их совместного распределения. Тогда

$$
\mathbf{P}\left\{\xi_{j} \equiv 0(\bmod 2), j=1, \ldots, m\right\}=\frac{1}{2^{m}} \sum_{z_{1}, \ldots, z_{m}= \pm 1} f\left(z_{1}, \ldots, z_{m}\right) .
$$

Доказательство. Утверждение леммы легко доказать по индукции, используя соотношения вида

$$
\begin{gathered}
f_{m-1}\left(z_{1}, \ldots, z_{m-1}\right) \stackrel{\text { def }}{=} f\left(z_{1}, \ldots, z_{m-1}, 1\right)+f\left(z_{1}, \ldots, z_{m-1},-1\right)= \\
=\mathbf{E} z_{1}^{\xi_{1}} \ldots z_{m-1}^{\xi_{m-1}}\left(1+(-1)^{\xi_{m}}\right)=2 \mathbf{E} z_{1}^{\xi_{1}} \ldots z_{m-1}^{\xi_{m-1}} I\left\{\xi_{m} \equiv 0(\bmod 2)\right\}, \\
f_{m-2}\left(z_{1}, \ldots, z_{m-1}\right) \stackrel{\text { def }}{=} f_{m-1}\left(z_{1}, \ldots, z_{m-2}, 1\right)+f_{m-1}\left(z_{1}, \ldots, z_{m-2},-1\right)= \\
=\mathbf{E} z_{1}^{\xi_{1}} \ldots z_{m-1}^{\xi_{m-2}}\left(1+(-1)^{\xi_{m-1}}\right) I\left\{\xi_{m} \equiv 0(\bmod 2)\right\}= \\
=4 \mathbf{E} z_{1}^{\xi_{1}} \ldots z_{m-2}^{\xi_{m-2}} I\left\{\xi_{m-1} \equiv \xi_{m} \equiv 0(\bmod 2)\right\},
\end{gathered}
$$

и т. д.

Пусть $g\left(z_{1}, \ldots, z_{N}\right)$ - производящая функция распределения вектора $X_{1}=\left(x_{1,1}, \ldots, x_{1, N}\right)$ :

$$
g\left(z_{1}, \ldots, z_{N}\right)=\mathbf{E} z_{1}^{x_{1,1}} \ldots z_{N}^{x_{1, N}}=\frac{1}{C_{N}^{s}} \sum_{1 \leq k_{1}<\ldots<k_{s} \leq N} z_{k_{1}} \ldots z_{k_{s}} ;
$$

тогда для производящей функции $g_{r}\left(z_{1}, \ldots, z_{N}\right)$ распределения суммы векторов $X_{1}, \ldots, X_{r}$, рассматриваемых как векторы с целочисленными неотрицательными координатами, справедливо равенство

$$
g_{r}\left(z_{1}, \ldots, z_{N}\right)=\mathbf{E} z_{1}^{x_{1,1}+\ldots+x_{r, 1}} \ldots z_{N}^{x_{1, N}+\ldots+x_{r, N}}=g^{r}\left(z_{1}, \ldots, z_{N}\right) .
$$

Из леммы 2 следует, что

$$
\mathbf{P}\left\{X_{1} \oplus \ldots \oplus X_{r}=0\right\}=\mathbf{P}\left\{x_{1, j}+\ldots+x_{r, j} \equiv 0(\bmod 2), j=1, \ldots, N\right\}=
$$




$$
\begin{gathered}
=\frac{1}{2^{N}} \sum_{z_{1}, \ldots, z_{N}= \pm 1} g_{r}\left(z_{1}, \ldots, z_{N}\right)=\frac{1}{2^{N}} \sum_{z_{1}, \ldots, z_{N}= \pm 1} g^{r}\left(z_{1}, \ldots, z_{N}\right)= \\
=\frac{1}{2^{N}} \sum_{z_{1}, \ldots, z_{N}= \pm 1}\left(\frac{1}{C_{N}^{s}} \sum_{1 \leq k_{1}<\ldots<k_{s} \leq N} z_{k_{1}} \ldots z_{k_{N}}\right)^{r}= \\
=\frac{1}{2^{N}} \sum_{t=0}^{N} C_{N}^{t}\left(\frac{1}{C_{N}^{s}} \sum_{1 \leq k_{1}<\ldots<k_{s} \leq N} z_{k_{1}}^{(t)} \ldots z_{k_{N}}^{(t)}\right)^{r},
\end{gathered}
$$

где $z_{j}^{(t)}=-1$, если $1 \leq j \leq t$, и $z_{j}^{(t)}=1$, если $t<j \leq N$. Следовательно,

$$
\begin{aligned}
& \mathbf{P}\left\{X_{1} \oplus \ldots \oplus X_{r}=0\right\}= \\
& \quad=\frac{1}{2^{N}} \sum_{t=0}^{N} C_{N}^{t}\left(\frac{1}{C_{N}^{s}} \sum_{j \geq 0}(-1)^{j} \cdot(1)^{s-j} C_{t}^{j} C_{N-t}^{s-j}\right)^{r}=\frac{1}{2^{N}} \sum_{t=0}^{N} C_{N}^{t}\left(\frac{C_{N, s, t}}{C_{N}^{s}}\right)^{r}
\end{aligned}
$$

И

$$
\begin{aligned}
\mathbf{P}\{F\} & \leq \sum_{2 \leq r \leq n} C_{n}^{r} \mathbf{P}\left\{X_{1} \oplus \ldots \oplus X_{r}=0\right\}= \\
= & \sum_{2 \leq r \leq n} C_{n}^{r} \frac{1}{2^{N}} \sum_{t=0}^{N} C_{N}^{t}\left(\frac{c_{N, s, t}}{C_{N}^{s}}\right)^{r}=\frac{1}{2^{N}} \sum_{t=0}^{N} C_{N}^{t} \sum_{2 \leq r \leq n} C_{n}^{r}\left(\frac{c_{N, s, t}}{C_{N}^{s}}\right)^{r}= \\
= & \frac{1}{2^{N}} \sum_{t=0}^{N} C_{N}^{t}\left[\sum_{0 \leq r \leq n} C_{n}^{r}\left(\frac{c_{N, s, t}}{C_{N}^{s}}\right)^{r}-C_{n}^{1}\left(\frac{c_{N, s, t}}{C_{N}^{s}}\right)^{1}-C_{n}^{0}\left(\frac{c_{N, s, t}}{C_{N}^{s}}\right)^{0}\right]= \\
& =\frac{1}{2^{N}} \sum_{t=0}^{N} C_{N}^{t}\left[\left(1+\frac{c_{N, s, t}}{C_{N}^{s}}\right)^{n}-n \frac{C_{N, s, t}}{C_{N}^{s}}-1\right] .
\end{aligned}
$$

\section{Список литературы}

1. Справочник по специальным функциям / под ред. Абрамовица М., Стиган И. - М.: Наука, 1979. - 831 с.

2. Alfers D., Dinges H. A normal approximation for Beta and Gamma tail probabilities // Z. Wahrscheinlichkeitstheorie verw. Gebiete. - 1984. V. 65. - P. 399-420. 
3. Балакин Г.В. Распределение ранга случайных матриц над конечным полем // Теория вероятн. и примен. - 1968. - Т. 13. Вып. 4. - С. 631-641.

4. Балакин Г. В. Системы булевых уравнений с искаженной правой частью при ограничениях на значения неизвестных и ошибок // В сб.: Труды по дискретной математике. - М.: ФИЗМАТЛИТ, 2008. - Т. 11. Вып. 1. С. 5-17.

5. Зубков А. М., Серов А.А. Оценки числа булевых функций, имеющих аффинные приближения заданной точности // Дискретн. матем. - 2010. T. 22. № 4. - C. 3-19.

6. Зубков А.М., Серов А.А. Полное доказательство универсальных неравенств для функции распределения биномиального закона // Теория вероятн. и примен. - 2012. - Т. 57. - Вып. 4.

7. Козлов М. В. О ранге матриц со случайными булевыми элементами // ДАН СССР. - 1966. - Т. 169. Вып. 5. - С. 1013-1016.

8. Коваленко И. Н. Об одной предельной теореме для определителей в классе булевых функций // ДАН СССР. - 1964. - Т. 161. Вып. 3. C. $517-519$.

9. Копытцев В.А. О числе решений систем линейных булевых уравнений в множестве векторов, обладающих заданным числом единиц // Дискретн. матем. - 2002. - Т. 14. Вып. 4. - С. 87-109.

10. Копытцев В.А. О числе решений системы случайных линейных уравнений в множестве векторов специального вида // Дискретн. матем. 2006. - Т. 18. Вып. 1. - С. 40-62.

11. Копытиев В.А., Михайлов В.Г. Теоремы пуассоновского типа для числа специальных решений случайного линейного включения // Дискретн. матем. - 2010. - Т. 22. Вып. 2. - С. 3-21.

12. Михайлов В.Г. Предельные теоремы для числа точек случайного линейного подпространства, попавших в заданное множество // Дискретн. матем. - 2003. - Т. 15. Вып. 2. - С. 128-137.

13. Сачков В.Н. Введение в комбинаторные методы дискретной математики. - М.: Наука, 1982.

14. Холл М. Комбинаторика. - М.: Мир, 1970. 